\title{
3D BUILDING MODELING BASED ON LOW ALTITUDE MAPPING SYSTEM
}

\author{
Zhou Yuan $^{\mathrm{a}} *$, Lin Zongjian ${ }^{\mathrm{b}}$, Gui Dezhu ${ }^{\mathrm{c}}$, Xie Feifei $^{\mathrm{d}}$ \\ ${ }^{a}$ Shandong University of Science and Technology,Qingdao,China.zhouyuan1318@126.com \\ ${ }^{\mathrm{b}}$ Chinese Academy of Surveying and Mapping,Beijing China.lincasm@casm.ac.cn \\ ${ }^{c}$ Development Research Center for Surveying \& Mapping, State Bureau of Surveying \& Mapping, Beijing, China. \\ guidezhu0930@163.com \\ d Wuhan University, Wuhan, China.xiefeifei_007@163.com
}

Commission I, ICWG I/V

KEY WORDS: Mapping, Photogrammetry, Texture, Triangulation, Building, System

\begin{abstract}
: a new way for the rapid development of the "digital city".

\section{INTRODUCTION}

As the needs of the digital city, converting the traditional $3 \mathrm{~d}$ spatial data to $2 \mathrm{~d}$ spatial data visualization already cannot satisfy the needs of people's stereo vision ${ }^{[1]}$. The existing $2 \mathrm{~d}$ GIS system stores a large number of data needed by threedimensional city modeling. Scholars had used assumed height and simulated texture to construct buildings, but this model is less realistic, insufficient in landscape information expression, with a large number of three dimensional space information such as height, body shape, texture, and other aspects of the details ignored. Also now modeling method can based on DSM and image, that is, combining the aviation image with DSM can generate landscape or city buildings' landscape model, but the landscape also lack of sense of reality, and it is hard to model $3 \mathrm{~d}$ object to a single model ${ }^{[2]}$.
\end{abstract}

The development and application of the mapping system with low altitude develops a new 3D city modeling technology means. 3D modeling method with low altitude has high efficiency, low cost, short cycle, strong maneuverability, convenient operation etc. This paper introduces related remote sensing data processing software and stereo mapping software of the low altitude mapping system. The paper also expounds quick acquisition of 3D city building texture based on the mapping system and 3D modeling by means of the software 3dmax. 3D city modeling combined low altitude mapping technology and the 3D modeling software 3dmax can open up

Now the theory and method of $3 \mathrm{~d}$ modeling of city buildings have made significant development, but there are still many problems to be solved. For example, modeling with $2 \mathrm{~d}$ vector data is not very good in the elevation accuracy; Building model from laser point cloud is deficient in morphology, and cost high; Traditional aviation stereo images has low automation. To sum up, $3 \mathrm{~d}$ building modeling with high precision, low cost, strong authenticity and high automation is the future development direction.

With the development of photogrammetry technology, the use of aviation stereo image pairs to determine the $3 \mathrm{~d}$ information becomes one of the mainstream directions in the reconstruction of $3 \mathrm{~d}$ features. This paper studies the low altitude mapping system taking building images in four directions. The images has wealthy information, providing the geometry information and real clear texture information of buildings, solving the problems of serious block in the dense building regions and serious lack of building surface texture information, possessing the advantages of high efficiency, low cost, short cycle, strong maneuverability, good safety and high images overlapping ${ }^{[3]}$, especially suitable for $3 \mathrm{~d}$ modeling of the city. Combined with $3 \mathrm{~d}$ modeling software 3 dmax, $3 \mathrm{~d}$ building model has clear texture and sense of reality.

\section{MODEL CONSTRUCTION AND TEXTURE ACQUISITION METHOD}

At present, the main data source of digital city model is space images, aviation image and laser scanning data,etc. There are periodic problem and low resolution for space images; $3 \mathrm{~d}$ laser point cloud data can directly reconstruct three-dimensional model of the buildings, but this model lack of building texture and need expensive equipment, to some extent, which restricting its application scope; Aviation images have the advantages of getting fastly and efficiently and more information, but the weather conditions influence data acquisition largely, in building concentration areas there is serious block phenomenon and it's hard to acquire the building facade texture from the images, which are current weaknesses of the aviation image ${ }^{[4]}$. At the same time, the emerging technology of low altitude mapping system not only has traditional aerial photogrammetry advantages, but also make up it's shortages, especially suitable for $3 \mathrm{~d}$ modeling of the city.

In the $3 \mathrm{~d}$ digital city construction, generally using the model added texture technology achieves the purpose of reproduce $3 \mathrm{~d}$ city scene. The traditional building texture acquisition basically has the following two kinds of means: the first, using common digital camera closely get building texture, but this method is highly affected from the seasons; The second, the helicopter video camera get building texture, this kind of method in a certain flight level can take images which has certain overlapping degree, but the cost is too high ${ }^{[5]}$. The low altitude mapping system developed by Chinese Academy of Surveying and Mapping, using low flying platform and wide angle camera

\footnotetext{
* Corresponding author- Zhou Yuan, master,Shandong University of Science and Technology ,China.zhouyuan1318@ 126.com.
} 
conduct aerial photography for the buildings around four directions and the top, getting all-round information of three dimensional space objects, and image resolution achieving $\mathrm{cm}$ level. The images after facade correction and well deal with light, can satisfy the requirements of $3 \mathrm{~d}$ building modeling of digital city. Low altitude mapping system is a new system of low altitude high resolution remote sensing image data quick acquisition $^{[6]}$.

\section{THE LOW ALTITUDE MAPPING SYSTEM AND 3D MODELING}

The low altitude mapping system integrated the hardware and software technology of the spacecraft, sensor system and data processing software, developing a complete set of low altitude mapping production operation flow, as shown in figure 1 with the whole flow chart of $3 \mathrm{~d}$ modeling. For combined camera, using the MCC software developed by the project team of Chinese Academy of Surveying and Mapping finishes the camera calibration and image mosaics. Using the interior orientation element and distortion parameters obtained from the single-phase camera calibration and equation (1) complete single image distortion correction; With the help of "relative exterior orientation element" initial value from combined camera calibration and feature matching for overlap image area, it can get precise stitching parameters, then using this parameters complete the virtual image mosaics. Essentially though proper mathematical model oblique CCD images from multi-lens can be converted to a virtual image with the same projective center, the same image plane, the same principal distance $^{[7]}$.

$$
\left.\begin{array}{l}
\Delta x=\left(x-x_{0}\right)\left(k_{1}+k_{2} r^{2}+k_{3} r^{4}\right)+p_{1}\left[r^{2}+2\left(x-x_{0}\right)^{2}\right]+2 p_{2}\left(x-x_{0}\right)\left(y-y_{0}\right) \\
\Delta y=\left(y-y_{0}\right)\left(k_{1}+k_{2} r^{2}+k_{3} r^{4}\right)+p_{2}\left[r^{2}+2\left(y-y_{0}\right)^{2}\right]+2 p_{1}\left(x-x_{0}\right)\left(y-y_{0}\right)
\end{array}\right\}
$$

Where: $\Delta x, \Delta y=$ change of image point;

$\left(x_{0}, y_{0}\right)=$ principal point of photograph coordinate,

$f$ =focal length;

$(x, y)=$ image coordinates;

$r=\sqrt{\left(x-x_{0}\right)^{2}+\left(y-y_{0}\right)^{2}}$ the distance from image point to principal point;

$k_{0}, k_{1}, k_{2}=$ radial distortion coefficient;

$p_{1}, p_{2}=$ tangential distortion coefficient .

Using single image or mosaic images, the existing automatic aerial triangulation measuring software MAP-AT (ModernAerial Photogrammetry Auto Triangulation) studied by the project team, full-automatically processes thousands of area array camera images obtained in low altitude flight and fullautomatically completes the aerial triangulation measurement. Practice proves that MAP-AT solves the existing limitation of large scale mapping with low altitude mapping system, very suitable for low altitude mapping production ${ }^{[8]}$.

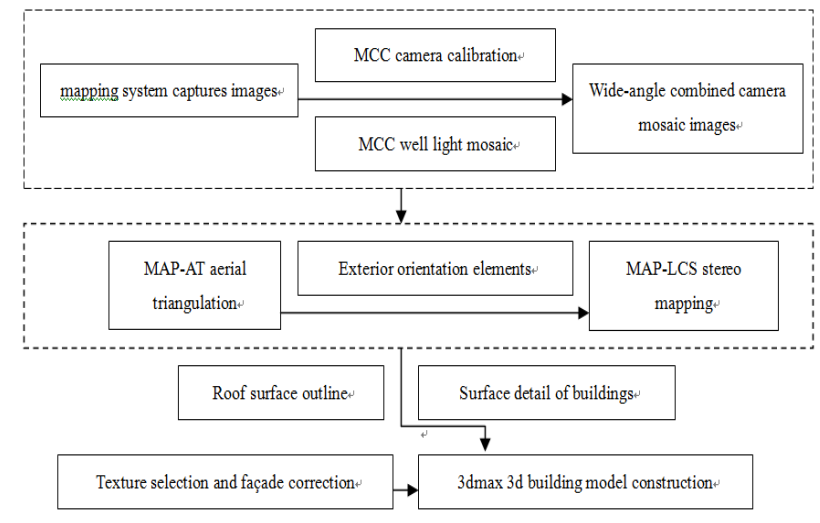

Figure 1. 3d modeling flow chart

Based on MAP-AT aerial triangulation results, a new stereo mapping software MAP-LCS developed by project team is used to stereo mapping. Map-LCS is a digital photogrammetry stereo processing workstation with friendly interface. By establishing stereo model, in the help of the stereo model and the pedal or handwheel, with stereo glasses MAP-LCS measures the roof edge line of the city's buildings, and the measured 3d vector data is real-time displayed in AutoCAD, realizing the acquisition and edit integration. The measured features have the attributes of feature code, layer, linetype, line width. According to the flight routes, setting up quantity stereo image pairs, switching stereo model automatically, MAP-LCS collects features' elements continuously on real-time nuclear line images, improving the working efficiency, reducing the workload of mosaic.

For the facade texture correction and more building surface details measurment, the buildings' facade texture processing software developed by project team can be used. Where, the facade of the building correction is completed according to the geometric constraint conditions of vertical, parallel, isometric and collinearity, etc; If the coordinates of two points in the image are $\boldsymbol{P}_{1}\left(X_{1}, Y_{1}, Z_{1}\right)$ and $P_{2}\left(X_{2}, Y_{2}, Z_{2}\right)$, then the length is $\mid P_{12}=\sqrt{\left(X_{1}-X_{2}\right)^{2}+\left(Y_{1}-Y_{2}\right)^{2}+\left(Z_{1}-Z_{2}\right)^{2}}$. If the actual distance of the two points is known, then the image scale $\lambda$ can be calculated. Similarly, for any actual distance D in the image, as long as the corresponding image length was measured, the actual distance D can be calculated from the equation (2). Thus it offers texture details size for 3 dmax modeling.

$$
D=\lambda d
$$

$3 d \max$ is the most popular, the most widely used $3 \mathrm{~d}$ software ${ }^{[1]}$. This paper using $3 d \max$ software as a tool, combining with $3 \mathrm{~d}$ roof vector data measured by stereo mapping software MAPLCS and the collected facade texture, researches the threedimensional modeling method. The experimental results show that this method is simple, reliable and $3 \mathrm{~d}$ result is lifelike and beautiful. 


\section{THE EXPERIMENT}

The $3 \mathrm{~d}$ modeling technology based on the low altitude mapping system is well proved in pingdingshan demonstration zone in Henan province. Application demonstration area of pingdingshan city put Henan city building college as the center. The total area is $2.5 \mathrm{~km} 2$, about $1.5 \mathrm{~km}$ long from east to west, and 1.8 kilometers width from north to south. The buildings in Henan city building college have more style, suitable for validating $3 \mathrm{~d}$ city model building ability based on low altitude wide-angle combined camera.

According to the distribution of building area, using the flying direction of east and west, navigation high $450 \mathrm{~m}$, heading overlap $65 \%$, lateral overlap $40 \%$ and the 4 wide-angle cameras combined SWDC obtained for the images of this demonstration area. The project team members get 226 four mosaic images with distortion corrected after image mosaic processing for experimental area. Based on the mosaic images and initial position elements provided by the field, the software MAP-AT conducts full-automatic aerial triangulation. Then based on aerial triangulation results, low altitude mapping system software MAP-LCS gets line graph of the experimental image. During this period, some checkpoints of this demonstration area are measured with the MAP-LCS, the measurement results are shown in table 1. From the table, it can be seen that the checkpoint precision can reach $\mathrm{cm}$ level.

\begin{tabular}{|c|c|c|c|c|}
\hline Dots & Type of data & $X$ & $\mathrm{Y}$ & $\mathrm{Z}$ \\
\hline $\begin{array}{c}101 \\
1\end{array}$ & $\begin{array}{l}\text { Validation } \\
\text { data } \\
\text { Lcs } \\
\text { measuring } \\
\text { data } \\
\text { error }\end{array}$ & $\begin{array}{c}424401.64 \\
8 \\
424401.62 \\
9 \\
-0.019\end{array}$ & $\begin{array}{c}3739012.57 \\
2 \\
3739012.60 \\
2 \\
0.03\end{array}$ & $\begin{array}{c}116.61 \\
1 \\
116.61 \\
5 \\
0.004\end{array}$ \\
\hline 303 & $\begin{array}{l}\text { Validation } \\
\text { data } \\
\text { Lcs } \\
\text { measuring } \\
\text { data } \\
\text { error }\end{array}$ & $\begin{array}{c}423750.15 \\
3 \\
423750.16 \\
2 \\
0.009\end{array}$ & $\begin{array}{c}3738876.77 \\
5 \\
3738876.75 \\
7 \\
-0.018\end{array}$ & $\begin{array}{c}136.23 \\
8 \\
136.23 \\
3 \\
-0.005\end{array}$ \\
\hline 313 & $\begin{array}{l}\text { Validation } \\
\text { data } \\
\text { Lcs } \\
\text { measuring } \\
\text { data } \\
\text { error }\end{array}$ & $\begin{array}{c}423787.33 \\
7 \\
423787.32 \\
6 \\
-0.011\end{array}$ & $\begin{array}{c}3739088.93 \\
8 \\
3739088.90 \\
9 \\
-0.029\end{array}$ & $\begin{array}{c}136.27 \\
9 \\
136.29 \\
7 \\
0.018\end{array}$ \\
\hline 405 & $\begin{array}{l}\text { Validation } \\
\text { data } \\
\text { Lcs } \\
\text { measuring } \\
\text { data } \\
\text { error }\end{array}$ & $\begin{array}{c}423855.61 \\
4 \\
423855.61 \\
6 \\
-0.002\end{array}$ & $\begin{array}{c}3738698.08 \\
6 \\
3738698.07 \\
6 \\
-0.01\end{array}$ & $\begin{array}{c}137.37 \\
4 \\
137.36 \\
8 \\
-0.006\end{array}$ \\
\hline 501 & $\begin{array}{l}\text { Validation } \\
\text { data } \\
\text { Lcs } \\
\text { measuring } \\
\text { data } \\
\text { error }\end{array}$ & $\begin{array}{c}423938.21 \\
8 \\
423938.21 \\
1 \\
-0.007\end{array}$ & $\begin{array}{c}3739012.20 \\
2 \\
3739012.20 \\
6 \\
0.004\end{array}$ & $\begin{array}{c}130.90 \\
2 \\
130.90 \\
8 \\
0.006\end{array}$ \\
\hline 602 & $\begin{array}{l}\text { Validation } \\
\text { data } \\
\text { Lcs } \\
\text { measuring } \\
\text { data } \\
\end{array}$ & $\begin{array}{c}424015.57 \\
8 \\
424015.54 \\
9 \\
-0.029 \\
\end{array}$ & $\begin{array}{c}3738944.37 \\
3738944.36 \\
4 \\
-0.006\end{array}$ & $\begin{array}{c}131.72 \\
6 \\
131.71 \\
7 \\
-0.009 \\
\end{array}$ \\
\hline
\end{tabular}

\begin{tabular}{c|c|c|c|c}
\hline & error & & & \\
\hline 604 & Validation & 424033.35 & 3738792.10 & 135.36 \\
& data & 8 & 9 & 135.36 \\
& Lcs & 424033.36 & 3738792.06 & 5 \\
& measuring & 4 & 1 & 0.005 \\
& data & 0.006 & -0.048 & \\
& error & & & \\
\hline 801 & Validation & 424167.97 & 3739012.22 & 122.55 \\
& data & 6 & 1 & 7 \\
& Lcs & 424167.95 & 3739012.17 & 122.54 \\
& measuring & 9 & 6 & 9 \\
& data & -0.017 & -0.045 & -0.008 \\
& error & & & \\
\hline
\end{tabular}

Table 1. checkpoint precision table (unit: $\mathrm{m}$ )

From the the accuracy of field checkpoint above, the data of this experiment can be used to mapping with large scale (this experiment is 1:1000). Mapping software is used to make vector line data to 226 images. In the process of stereo mapping, it puts the vector with three dimensional attribute into CAD in real time, getting *. DWG files. The work interface is shown in figure 2. Figure 3 is the effect of putting the corresponding *. DWG files into $3 \mathrm{~d}$ modeling software $3 d \max$ using the library of the demonstration zone as an example. From the left and front view it can be seen that the vector possess data $3 \mathrm{~d}$ information. using the vector the library model is builded in the software 3dmax. For the model texture, using the building façade processing software developed by the project team correct the oblique facade images and measure the details of the building size, then the software photoshop can used to cut out texture for modeling. The effect of model added texture information is shown in figure 4 .

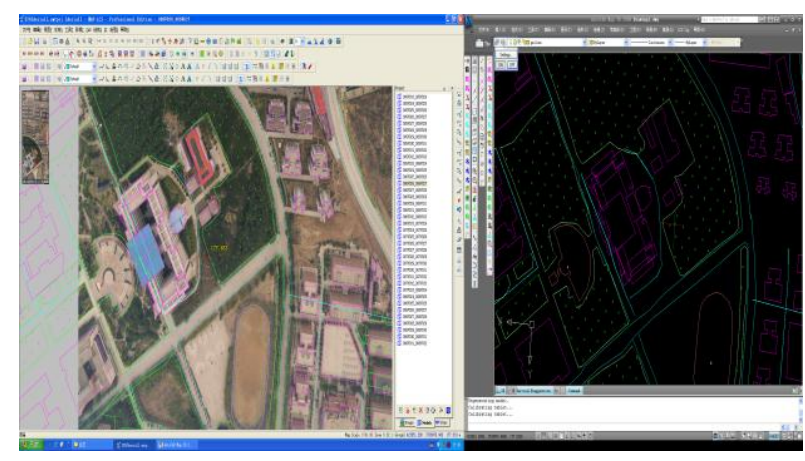

Figure 2. MAP-LCS stereo mapping for $3 \mathrm{~d}$ vector data

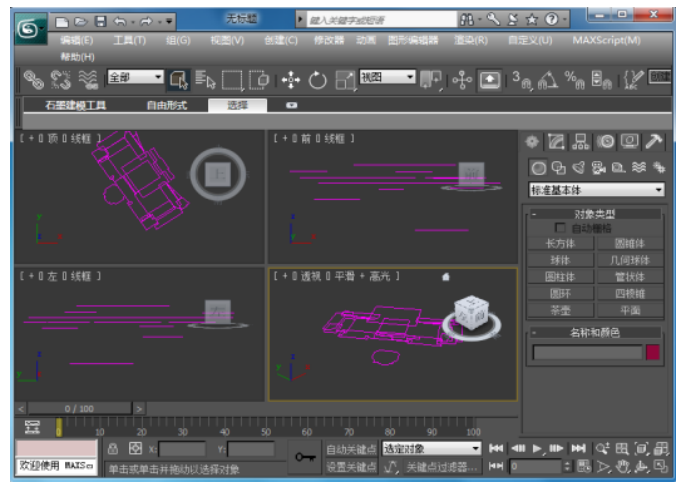

Figure 3. * DWG files into $3 \mathrm{dmax}$ 


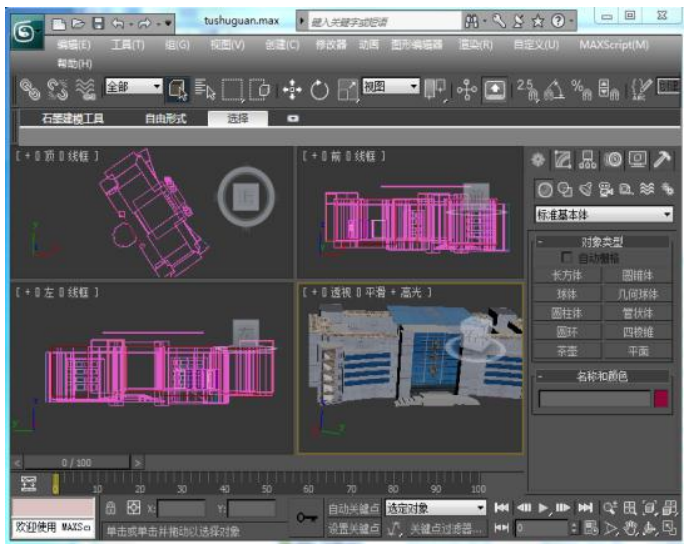

Figure 4. Modeling effect in the $3 \mathrm{dmax}$

The modeling proceeded for all buildings in pingdingshan demonstration zone, plus DEM information and projective image of the area, realizing $3 \mathrm{~d}$ real scene reconstruction of the buildings. Overall effect is shown in figure 5 .

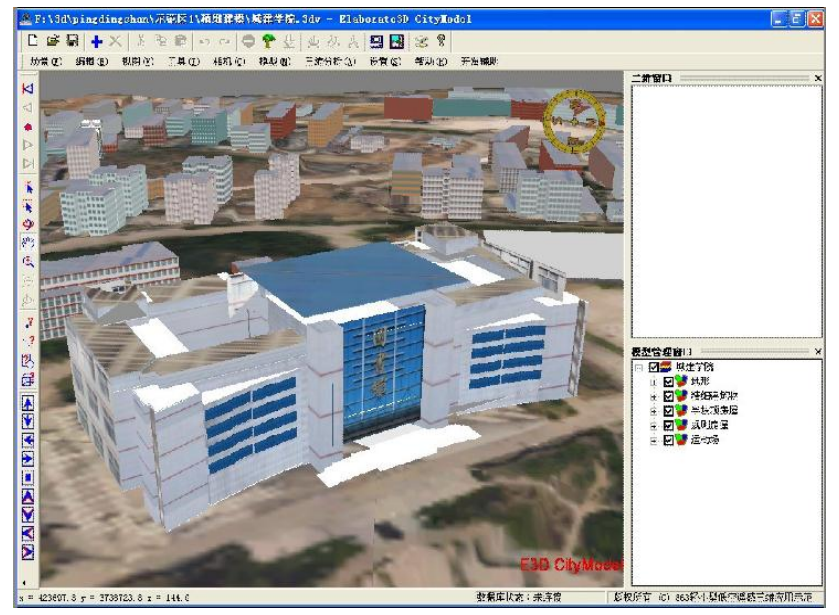

Figure 5. 3d scene reconstruction in pingdingshan

Experiments show that the low altitude mapping system has the characteristics of flexible, mobile, rapid, etc. Through the reasonable route planning, it can get the top face of buildings and the texture of four laterals at the same time, effectively solving the problem of texture acquisition of complex city buildings for $3 \mathrm{~d}$ modeling, and it has a good application prospect. But in pingdingshan application demonstration area, it also exists a problem: in order to get good building side texture and easy to measure the lateral details of the buildings, operators need to adjust the angle between the cameras and further reduce the flight height, at the same time, it can acquire building texture in all position through the flight direction of east-west and north-south.

\section{CONCLUSION}

Photogrammetry technology will develop continuously towards advance along the direction of digital, automatic and visual, and cross development with other disciplines will also offer new development prospects. This paper discusses the low altitude mapping technology and remote sensing images with high overlap and high resolution which will be good in application of $3 \mathrm{~d}$ modeling and information extraction. Combined $3 \mathrm{~d}$ modeling software (such as: 3 dmax), 3d city modeling can open up a new way with the rapid development for the "digital city", and make the contribution to the modernization construction of our country.

\section{REFERENCE}

[1]. Peng Fangyuan, Zhou qisheng, Xiang Changgan. 2011. 3d modeling method research based on the aerial data [J]. Science of Surveying and Mapping, 34 (2),pp63-65.

[2]. Gao Yang, Li Jin. 2008. 3d modeling method research for digital city [J] City Survey, 2008 (2),pp $11-13$.

[3]. Cui Hongxia, Lin Zongjian, sun Jie, etc. 2005.3d modeling method research for large overlapping UAV remote sensing image [J]. Science of Surveying and Mapping, 30(2),pp3638.

[4]. Li Yongquan, Han Wenquan, Huang Zhizhou. 2010.The comparison and analysis of $3 \mathrm{~d}$ digital city modeling methods [J]. Modern Surveying and Mapping, 33 (2),pp3335.

[5]. Liu Zhaoqin, Sun Chengzhong, sun Jie. 2006 .The method of three-dimensional city building texture quick acquisition based on the low altitude UAV remote sensing system [J]. Remote Sensing Information, 2006 (5),pp47-49.

[6].Cui Hongxia,Lin Zongjian,Yang Hong. 2008.Multi-angle photogrammetry of low altitude UAV digital images [J].Opto-Electronic Engineering,2008(7),pp73-78.

[7].Cui Hongxia,Lin Zongjian,Meng Wenli. 2009.Relative selfcalibration of large area array digital cameras [J]. OptoElectronic Engineering,2009(6),pp81-85.

[8].Lin Zongjian,Su Guozhong,Zhi Xiaodong. 2010.Low altitude UAV borne double camera mapping system [J]. Geospatial Information,2010(4),pp1-3. 